\title{
Donald Trumps Medien
}

\author{
Niels Werber
}

\section{„Potus als Twitterer"}

Am 20. Januar 2017 hat Donald Trump das Amt des 45. Präsidenten der Vereinigten Staaten von Amerika übernommen. An diesem Tag versendet Barack Obama seinen letzten Tweet vom Twitter-Account des President of the United States, @ potus: „I'm still asking you to believe - not in my ability to bring about change, but in yours. I believe in change because I believe in you." Der Account wird noch am selben Tag als @ potus44 von Twitter archiviert. @Potus steht ab dem 21. Januar 2017 dem neuen Präsidenten, Donald Trump, zur Verfügung. Von diesem Account wird an diesem 21.01.17 folgender Tweet abgesetzt: „January 20th 2017, will be remembered as the day the people became the rulers of this nation again." Derselbe Tweet wurde kurz zuvor vom Account @ realDonaldTrump abgesetzt. Damit wird eine kurzlebige Praxis etabliert: Der präsidiale @potus Account setzt Mitteilungen ab, die bis ins letzte Zeichen identisch mit Tweets sind, die vorher @ realDonaldTrump getwittert hat. Ab Februar 2017 habe ich keine Beispiele mehr dafür finden können, dass@potus und @ realDonaldTrump in dieser Form die gleichen Botschaften versenden. Eine neue Praxis ist etabliert worden: @potus retweetet nun jeden Tweet, der von@ @realDonaldTrump versendet wird. @potus ist ein Retweetbot. Das Amt dient als Amplifier der Twitter-Reichweite des Milliardärs, der schon seit März 2009 einen Account unterhält und am 4. Mai 2009 seinen ersten Tweet abgesetzt hat. Seitdem geht es rasant aufwärts. Am 29. Oktober 2009 zählt Trump nahezu 500.000 Follower, aufgrund der schnellen Steigerung seiner Resonanz wird (von der Statistikplattform https://twittercounter.com/realDonaldTrump) prognostiziert, binnen 179 Tagen die Zahl von einer

N. Werber $(\square)$

Siegen, Deutschland

E-Mail: werber@germanistik.uni-siegen.de 
Million Follower zu erreichen. Auch wenn es nicht unbedingt der „,best Account to follow on Twitter“ (03.06.2013) sein mag, wie Trump das mehrfach für sich reklamiert, steht doch fest: Trump ist ein Twitterer, der Präsident wird. Am Tag vor der Amtsübernahme (19.01.2017) folgen seinem Account @ realDonaldTrump 20.322.176 Twitter-Follower, eine veritable Multitude menschlicher und nicht-menschlicher Agenten, Anhängerïnnen und Gegnerïnnen. Zu dieser durchaus heterogenen Menge von Abonnentïnnen des Accounts, die alles andere als eine Gefolgschaft darstellt, später mehr auch zum Politischen einer Medienpraxis, die heute geradezu selbstverständlich, etwa von Peter Strohschneider in seinem Essay „POTUS als Twitterer“, als „populistische Textpraxis“ bezeichnet wird (Strohschneider 2018: 61).

Zunächst ist Trumps Twittern aber erst einmal eine Praxis, die vor allem auf Popularität zielt und Popularität auch nachweisbar erreicht. Diese Popularität von @ realDonaldTrump lässt sich messen und vergleichen - und zwar sehr genau und mit allen anderen Accounts, wer auch immer sie betreiben mag. Die Popularität im Sinne messbarer Beachtungserfolge, auf die es hier ankommt, setzt zuerst und vor allem auf Quantitäten - unterläuft also alle tradierten high/low-Unterscheidungen, Qualitätsstandards, Regeln der Meinungsäußerung im öffentlichen Raum, Kohärenzansprüche usw. Dies lässt sich sehr gut im Jahr 2009 beobachten, dem Jahr, in dem Donald J. Trump sich einen Twitter-Account zulegt.

\section{Trumps und Twitters Popularität}

Am 17.04.2009 hat der junge Schauspieler und damalige Lebensgefährte von Demi Moore, Ashton Kutcher, gegen $C N N$ den Wettbewerb gewonnen, wer als erster eine Million Follower auf Twitter erreicht. Dass Kutcher gewinnt, zeigt ganz schön, dass die Popularisierungsbedingungen auf Twitter andere sind als die der traditionellen Massenmedien. Es ist fraglich, ob Kutcher $C N N$ bei den Einschaltquoten schlagen würde, würde er einen Nachrichtensender gründen. Ebenfalls bliebe unwahrscheinlich, ob er im Falle einer Zeitungsgründung hinsichtlich der Abonnementzahlen erfolgreich mit der New York Times konkurrieren könnte. Kutchers Twitter-Sieg illustriert, dass es auf der Plattform vor allem um Beachtung geht, die beispielsweise in Follower-Zahlen zu messen ist, und nicht um all die unzähligen Unterschiede, die zwischen den Tweets von Ashton Kutcher und den Nachrichten bestehen, die $C N N$ sendet.

Das Wettrennen eines Popstars gegen einen der wichtigsten Nachrichtensender der Welt hat aber vor allem ganz entscheidend dazu beigetragen, die zuvor unbekannte Mikroblogging-Plattform eines Start-ups zu popularisieren (vgl. Paßmann 2018: 290). „Twitter race: Ashton Kutcher trumps CNN with one million followers“, lautet eine Schlagzeile aus dem April 2009 (Telegraph vom 17.04.2009). Kurz nach dieser Erfolgsmeldung mit ihrem Seitenhieb auf die etablierten Massenmedien schickt der Account @ realDonaldTrump seinen ersten Tweet in die Welt. Trump startet nicht mit dem Thema CNN, sondern mit CBS und zwar mit dem Hinweis auf seinen Auftritt in einer sehr populären late night 
show: „Be sure to tune in and watch Donald Trump on Late Night with David Letterman as he presents the Top Ten List tonight“ (04.05.2009)! Trump präsentiert an diesem Abend in der Late Show auf CBS mitten in der Bankenkrise zehn mehr oder minder originelle Finanztipps von ,buy golf courses“ (natürlich in seinen Resorts) über ,sent me 29.99 \$ to get a tip und visit the Trump casino in Atlanta“ bis zu seiner „No. 1: marry and divorce me“. ${ }^{1}$ Der bemühte Witz besteht darin, in der Finanzkrise das Geld in Richtung Trump fließen zu lassen, der im Jahre 2009 zum vierten Mal bankrottiert und die Insolvenz seines Unternehmens Trump nach „Chapter 11“ zu restrukturieren sucht (Berke 2015).

Im Rückblick ist an diesem ersten Tweet interessant, dass Trump weit davon entfernt ist, das Massenmedium Fernsehen $\mathrm{zu}$ schmähen und seine Social Media-Präsenz pauschal gegen die Fake News Media in Stellung zu bringen, wie es nach Auskunft Peter Strohschneiders typisch für Donald Trump sein soll (Strohschneider 2018: 61, 71 f.). Der erste Tweet, der gegen die Fake News wettert, stammt erst vom 10. Dezember 2016 und scheint wieder eher auf etwas plumpe Art witzig gemeint zu sein als die Offensive eines „Medienkriegs“ einzuleiten (ebd.: 72). Trump twittert: „Reports by @ CNN that I will be working on The Apprentice during my Presidency, even part time, are ridiculous \& untrue - FAKE NEWS!“ Erst ein Jahr später wird die Bezeichnung „FAKE NEWS“ in einem Trump-Tweet zur Parole einer Diffamierungskampagne eines amerikanischen Präsidenten gegen die Nachrichtenmedien seines Landes: „FAKE NEWS A TOTAL POLITICAL WITCH HUNT“ (10.01.2017)!

Was Trump in seinem ersten und durchaus exemplarischen Tweet unternimmt, ist der Versuch, seinen Account über die Referenz einer populären TV-Show eines prominenten TV-Hosts zu popularisieren, überdies mit dem Hinweis auf das selbst sehr populäre Format einer Top-Ten-Liste. Parasitäre Popularität könnte man es nennen, wenn dieser erste Tweet Follower, Kommentare und Favs dadurch generiert, dass er alle Late Show- und Letterman-Fans adressiert. Denn es ist schwer auszumachen, ob die Twitter-Resonanz tatsächlich Trump gilt oder nicht doch David Letterman, seinem Gastgeber, dessen The Late Show im Jahr 2009 durchschnittlich 3,8 Mio. Zuschauer zugesehen haben, also über 3 Mio. mehr als Trump zu dieser Zeit Twitter-Follower zählt. Genau so hat Twitter zwei Wochen zuvor von der Popularität von $C N N$ profitiert, insofern der extrem reichweitenstarke Nachrichtensender über seinen Wettlauf mit Ashton Kutcher ausführlich berichtet hatte. Massenmedien sind für die Popularisierung Twitters entscheidend. Dass sie als Popularisierungsverstärker fungieren, hat sich bis heute nicht geändert. Trump verweist in seinen Tweets bis April 2019510 mal auf CNN, 102 mal auf CBS, 953 mal auf NBC, 1480 mal auf Fox und einige hundertmal auf Washington Post und New York Times. Umgekehrt werden seine Tweets in diesen Medien zitiert und kommentiert, worauf Trump wiederum häufig per Twitter hinweist. Aller erklärten Feindschaft zwischen den „fake news media“ (119 mal) und dem Präsidenten

\footnotetext{
${ }^{1} \mathrm{https}: / /$ www.youtube.com/watch?v=Saznopjn5WY\&t=1s (11.03.2019).
} 
steigern Trumps Twitter-Account und diese Massenmedien wechselseitig ihre Popularität. Die Zahl der Abonnentïnnen steigt auf beiden Seiten.

Zurück zu Trumps Top Ten, die sein erster Tweet annonciert. Ganz oben auf der Liste, auf Platz 1, platziert Trump seinen Supertipp für den Weg zum Geld in der subprime crisis: „marry and divorce me“. Ganz oben steht er selbst. „Was an der Spitze einer Rangliste von Dingen steht, die Menschen besitzen, (ver-) wünschen oder - im Falle von Aussagen - sich als Meinung zurechnen, gehört zur populären Kultur im weitesten Sinne“ (Hecken 2006: 87). Trump reklamiert Popularität in einer Form, die Vergleichbarkeit erlaubt und so Rankings ermöglicht: Platz eins liegt vor Platz zwei, worum es auch immer gehen mag. Genauer betrachtet, handelt es sich bei Trumps Top Ten um eine „auktoriale Liste“ und nicht um eine ,anonyme“, algorithmisch generierte Liste (Schaffrick 2016: 110), insofern Trumps Top Ten nicht der Reihenfolge empirisch gemessener und ausgewerteter Popularität entspricht, wie dies etwa bei den Top Ten-Listen der Musikcharts und Hitparaden der Fall ist. Es ist eher eine Liste, wie sie Literatur-, Musik- oder Filmkritikerïnnen zusammenstellen, wenn sie die besten zehn Romane, Singles oder Spielfilme aller Zeiten oder auch der Saison auswählen und in eine Rangfolge bringen; auch dies ist eine genuin populärkulturelle Praktik. Obgleich die Auswahl der Items und ihre Anordnung in Trumps auktorialer Top Ten-Liste also ganz offensichtlich allein ,individuell zurechenbar“ ist, versäumt es Trump nicht, sich selbst ganz oben zu positionieren, ganz so, als komme diesem Platz 1 die gleiche Bedeutung zu wie dem Platz 1 in einem Ranking empirischer Beachtungsmessung. Auch dies ließe sich als parasitäre Popularität bezeichnen. Der Platz 1 einer auktorialen Liste profitiert von der Popularität, die den metrischen Verfahren der Beachtungsmessung gemeinhin zukommt.

In Trumps Timeline kann man beides beobachten: Den Verweis auf solche Ranglisten, die aufgrund von quantitativen Messverfahren Zählergebnissen ausstellen und Zahlen in Ziffern ausdrücken (Heintz 2018: 633), und die Aufstellung auktorialer Ranglisten, je nachdem ob Trump sich gut platziert vorfindet oder ob er selbst sich gut platzieren muss. Der Form nach unterscheiden sich diese Listen nicht: Es sind die Top Ten oder die Top Five. Auch nach anderen Auftritten in Late Night Shows bezeugt Trump auf Twitter sein Interesse an quantitativ erwiesenen Beachtungserfolgen. „Ratings were great! A big win“, heißt es in einem Tweet vom 26. Januar 2016 über eine Miss Universum Wahl. Beispiele aus dem Wahlkampf: „The media refuses to talk about the three new national polls that have me in first place. Biggest crowds ever - watch what happens" (21.10.2016)! Und: „@CNBC continues to report fictious poll numbers. Number one, based on every statistic, is Trump (by a wide margin). They just can't say it" (28.10.2015)! Trump macht seine Popularität immer wieder zum Thema seiner Tweets: „Wow, ratings are just out", twittert er am 22.01.2017 über die beste und größte Inauguration aller Zeiten.

Solche Verlautbarungen sind typisch für eine ganze Serie von Tweets, die alle Trumps Popularität behaupten, belegen, inszenieren und weiter popularisieren - und zwar sehr häufig im Sinne eines Rankings, also typisch für jene Verfahren der Erzeugung und Visibilisierung von Differenz, die Steffen Mau zufolge 


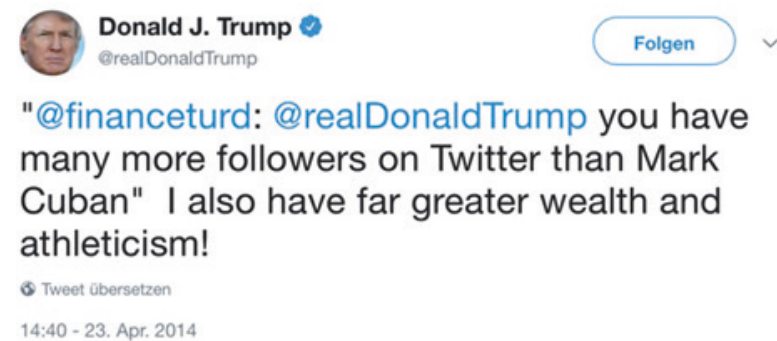

Abb. 1 Tweet von @ realDonaldTrump (23.04.2014)

„die Popularität von Politikern oder Popstars“ gleichermaßen hierarchisieren (Mau 2017: 71). Trumps Tweets fokussieren immer wieder auf „metrische Wertigkeitsordnungen“, deren Zahlen einen „Wert" nicht nur anzeigen, sondern auch zuteilen (ebd.: 261 f.). Die Tweets eröffnen immer wieder neue „Vergleichsdimensionen“ (ebd.: 262), in denen Trump exzelliert. Er ist smarter, reicher, athletischer und populärer als alle anderen, er hat die besseren Hotels, die schöneren Golfclubs, die beliebtere Show, die größere Zahl von Twitter-Follower (Abb. 1). Trump is trumping everybody else. Am 14. September 2014 twittert er: „Money was never a big motivation for me, except as a way to keep score. The real excitement is playing the game!"“ Geld lässt sich - wie die Zahl der Follower, Retweets, Favs oder Likes - leicht und genau messen, sodass sich angeben lässt, wer mehr und wer weniger hat. Wie bei der Quantifizierung des Sozialen insgesamt, dienen die von Trump aufgerufenen Metriken der „Universalisierung“ und „Inszenierung des Wettbewerbs“ sowie der Überführung qualitativer Differenzen in „quantitative Unterschiede“ (ebd.: 17). Die quantitativ messbare Popularität, die in „Ranglisten“ überführt wird und auf die es Trump ankommt, dient durchaus nicht einer ,Valorisierung des Besonderen“, sondern dem Nachweis eines kleinen, aber nachweisbaren Abstands, der Trump ,prozentual“ von anderen „Stars“ (Heintz 2018: 639) unterscheidet.

Trumps Tweets vollziehen dies nicht nur, sie reflektieren auch die Bedingungen der Beachtungserzeugung durch scores, charts, ratings, rankings usw. Dies gilt ausdrücklich nicht nur für seine Shows oder Unternehmen, sondern auch für Trumps Rolle als Politiker. Trumps ,politische“ Tweets belegen genau wie alle anderen das Gewicht, das der Popularität schlechthin und insbesondere der populären Inszenierung quantitativ messbarer Popularität in Rankings oder Ratings zukommt. „Polls looking great! See you soon“, schreibt er am 7. November 2016. Oder „Any negative polls are fake news, just like the CNN, ABC, NBC polls in the election“, am 6. Februar 2017. Oder Trump retweetet Fox \& Friends am 2. Juni 2017: „Wall Street hits record highs after Trump pulls out of Climate pact“. Populäre Politik ist messbar.

Bekanntlich hat Trump den popular vote gegen seine Konkurrentin Hillary Clinton verloren und sich geweigert anzuerkennen, dass sie knapp 3 Mio. Stimmen mehr für sich gewinnen konnte. Trump twittert zu seinem Sieg: „In addition 
to winning the Electoral College in a landslide, I won the popular vote if you deduct the millions of people who voted illegally“ (27.11.2016). Auch den рориlar vote habe er also in Wahrheit für sich entschieden, wenn man nur all die ,illegalen“ Wählerinnen und Wähler abzöge (vgl. Hecken 2017: 13). Damit noch einmal zurück zu Trumps Wahlerfolg und dem Tweet von@ potus vom 21. Januar 2017.

\section{Populismus}

„January 20th 2017, will be remembered as the day the people became the rulers of this nation again“" (20.01.2017). Vorher wurde das „Volk“ von korrupten Eliten eher betrogen als regiert; nun aber, mit Trumps Amtsübernahme, übt das „wahre Volk“ endlich wieder selbst die Staatsgewalt aus, so ließe sich dieser Tweet mit Jan-Werner Müller programmatisch als populistisches Programm reformulieren. Müller hat in einem instruktiven und sehr erfolgreichen Essay einen Vorschlag zur Definition unterbreitet, was Populismus ,eigentlich“ bzw. wer ,wirklich ein Populist" sei (Müller 2015: 28; Müller 2016: 11): Im Unterschied zu vielen ähnlichen Phänomenen von Extremistïnnen bis zu stammtischseligen Advokaten der Volkstümlichkeit seien Populismen anti-elitär und anti-pluralistisch zugleich (Müller 2015: 30). Der „Kernspruch aller Populisten“, so macht Müller seine Hypothese anschaulich, laute ,stets ungefähr so: ,Wir - und nur wir - repräsentieren das wahre Volk“" (Müller 2016: 26). Und diese Anmaßung spricht jeder anderen Partei und selbst dem vom Volk gewählten Parlament bzw. dem gewählten Staatsoberhaupt ab, das wahre Volk wirklich zu vertreten. Müllers Definition ist - aller berechtigten Kritik an ihrer Formalität (Manow 2019: 27 f.) zum Trotz hilfreich, denn sie scheidet alle nur möglichen Politikerinnen und Politiker aus, die derb oder volksnah auftreten, Probleme vereinfachen oder zuspitzen, leugnen oder mit absurden Mitteln zu lösen versprechen, jedoch ,keinen solchen moralischen Alleinvertretungsanspruch für sich reklamier[en]“ (Müller 2016: 26). Der in der Populismusforschung verbreitete Verzicht auf eine inhaltliche Bestimmung des Begriffs (Manow 2019: 27) zugunsten eines rein formalen Verständnisses (anti-pluralistisch, anti-elitär, vgl. Müller 2016: 26) erklärt womöglich den Erfolg des Vorschlags, der keine Unterscheidungen zwischen unterschiedlichen Regionen, politischen Systemen, Traditionen politischer Partizipation, Medienpraktiken, Ideologien, Ökonomien usw. erfordert.

In seinem Aufsatz „POTUS als Twitterer“ hat Peter Strohschneider diesen Beitrag Müllers ausführlich referiert und als Muster für seine Analyse der „spezifischen Textpraxis populistischer Politiken“ (Strohschneider 2018: 63) übernommen. Seine Twitter-Lektüren verdichtet Strohschneider zur These einer „Strukturhomologie zwischen populistischer Politik und einem bestimmten Typus netzschriftlicher Textpraxis“, insbesondere natürlich der „trumpschen Tweets“ (ebd.: 71). Trumps „,populistische Textpraxis“, so Strohschneider, konstituiere einerseits ein „homogenes Kollektiv“, quasi Müllers „wahres Volk“, und reklamiere fortwährend für sich und nur für sich, anders als die korrupten Eliten dieses 
wahre Volk zu repräsentieren (ebd.: 61, 68). Nimmt man Müllers Definition als Suchraster und durchmustert Trumps Timeline nach anti-elitären und anti-pluralistischen Tweets, dann wird man häufig genug fündig. Allerdings hat man dann keine Medienpraxis beschrieben, schon gar keine Twitter-spezifische Praktik, sondern eine semantische Analyse durchgeführt, die auch bei einer anderen Medienauswahl zu den gleichen Ergebnissen geführt hätte: Was Trump im TV sagt, in seinen Shows, in seinen Pressebriefings, in Zeitungsinterviews - das alles ließe sich auch nach der Müller-Formel ertragreich durchmustern. Man würde auch bei Grillo, le Pen, Wilders, Orbán oder Chavez fündig werden, um die Personen zu nennen, die als Motive eines populistischen Quartettspiels das Cover von Müllers Buch (2016) zieren. Weltweit ließen sich so schnell überzeugende Kandidatïnnen für die Etikettierung als Populistïn finden. Schwieriger wird es, aufgrund dieser Musterung spezifischer zu werden. Warum es im Falle Trumps zu einer „Strukturhomologie zwischen populistischer Politik und einem bestimmten Typus netzschriftlicher Textpraxis“ gekommen sein soll, erschließt sich so nicht, denn die populistische Politik im Sinne Strohschneiders bzw. Müllers findet man überall dort, wo Trump sich äußert, auf seinen Rallies, in den Massenmedien und eben auch in den sozialen Medien. Ich werde gleich selbst auf die Spezifität des Twitterns einzugehen suchen, aber um meine Beobachtungen zu konturieren, möchte ich zunächst Strohschneiders Thesen zu „POTUS als Twitterer“ referieren und kommentieren. Zwei zentrale Passagen seien hier im Zusammenhang zitiert:

\footnotetext{
„Die Trumpsche Tweet-Praxis unterstellt je schon alternativenloses Verständigtsein. Es geht ihr nicht ums Mitteilen und Verstehen, sondern um eine jenseits dessen liegende Teilhabe, um Partizipation. Darauf antwortet ein nicht reflexives, sondern im Gegenteil reflexhaftes Einverständnis, das als following (oder sharing) die zweideutigkeitslose Wirklichkeit einer homogenen Gefolgschaft integriert. Soziale Binnendifferenzierungen fehlen in ihr ebenso wie semantische Probleme. So wird ein Zusammenhang konstituiert, der weder einer Sprache der Distanz noch einer Sprache tatsächlicher Nähe zugeordnet werden könnte. Er ist in semantischer Hinsicht durch a-hermeneutisches Einverständigtsein gekennzeichnet und strukturell durch etwas, das man eine jenseits der Unterscheidung von Distanz und Nähe angesiedelte Abstandslosigkeit nennen möchte. Er wirkt als ein homogener Textraum, der all denjenigen, die ihn als follower liken, einen Erlebnismodus des phantasmatischen Darinnenseins ermöglicht. Insofern handelt es sich um eine Textlichkeit, die in dauererregter Exklamatorik ihre eigene medientechnische Vermitteltheit aus der Wahrnehmung bringt“" (Strohschneider 2018: 68 f.).
}

Kein einziger Tweet, den Strohschneider zitiert, entspricht dieser Beschreibung. Der Tweet „A great day at the White House!“ vom 31.07.2017 (ebd.: 68) beispielsweise erhält bis Dezember 201822.745 Retweets und 113.293 Likes - schaut man aber einmal in die Unmenge der Kommentare, dann sieht man sofort, dass weder Following, noch Liken oder Retweeten unbedingt Beispiele für ,reflexhaftes Einverständnis“ darstellen. Der Tweet findet zwar durchaus enthusiastische Zustimmung, trifft zugleich aber auch auf rigorose Ablehnung. Lobpreisungen und Hasstiraden wechseln sich ab. Die vielen Retweets werden genauso mit affirmativen Kommentaren versehen wie mit kritischen, mit invektiven oder absurden Anmerkungen oder gar mit Werbebotschaften unterschiedlichster Akteure, die von 
der Popularität des Accounts profitieren wollen und ihn so zugleich weiter popularisieren. Gerade das, was Strohschneider sharing nennt, fällt überaus ambivalent aus. Der Tweet wird zwar verbreitet und mit den eigenen Followers geteilt, doch wird er eben auch auf jedem Account neu gerahmt. Das sieht dann bei einer Trumpistin ganz anders aus als bei einem Trump-Kritiker (s. Abb. 2a, b).

Ich zitiere nun die zweite Passage aus Strohschneiders Aufsatz:

„Und allein das sharing dieser Rede als likender follower gewährt reale, vermittlungslose Teilhabe an dieser Realität. An die Stelle räumlich-zeitlicher, epistemischer und sozialpolitischer Differenzen und Kontingenzen tritt eine alle Vermitteltheiten wie Vorbehalte einebnende heiße, augenblickliche Gemeinschaftlichkeit, ein präsentistisches ,Hier ${ }^{`}$ und ,Jetzt " und ,Wir in unserer realDonaldTrump-Wirklichkeit" - dies aber nicht als religiöse Erfahrung oder als ästhetisches Ereignis, sondern als Instrument eines machtpolitischen Kalküls. Es ist ein Angriff auf alle Nicht-Follower. Ihnen fehle nämlich der access zu Trump und damit derjenige zur Wirklichkeit. Was sie zu sagen haben, entbehre jeglicher Geltung: yet they know nothing about me \& have zero access. \#FAKE NEWS! [...] Dies ist die Programmatik einer alles Intermediäre ausschaltenden, populistischen Unmittelbarkeit und Vorbehaltlosigkeit. Zugleich sollte aber deutlich geworden sein, dass der Textualitätstyp der Trumpschen Tweets selbst strukturell populistisch ist. Sie realisieren in bedrückender Ausschließlichkeit, was das Kurznachrichtenmedium strukturell schon ermöglicht: die Reduktion aller Urteilsbildung auf den anti-hermeneutischen Dual eines begründungsfreien like und dislike sowie die Einteilung der Welt in Freund und Feind, in Follower und alle anderen. Die Tweets konstituieren einen netzschriftlichen Kommunikationsraum, in dem die Gemeinschaft der Follower mit ihrem Präsidenten in abstandsloser Direktheit zusammenkommt. Man teilt sich in alternativlose Fakten: Verschiedenste Sachverhalte - am 12. September 2017 Präsidentschaft, Journalismus, Unwetter, Kindsgeburten, Diplomatie - unterliegen unterschiedslos einer unzweifelhaften, seriell unentwegt reproduzierten konsensuellen Bewertung. Man teilt ein gemeinsames gewisses Wissen, in dem sich ein je schon gegebenes Verständigtsein direkt manifestiert" (ebd.: 69 f.).

Dies ist unzutreffend: Es gibt weder eine Gemeinschaft der Follower in dem Sinne, dass die Twitter-Follower des @ realDonaldTrump-Accounts eine immer schon mit Ihrem Führer einverstandene Gefolgschaft Trumps darstellen würden, noch gibt es eine Feinderklärung an die Nicht-Follower. Die Follower zerfallen vielmehr selbst

a

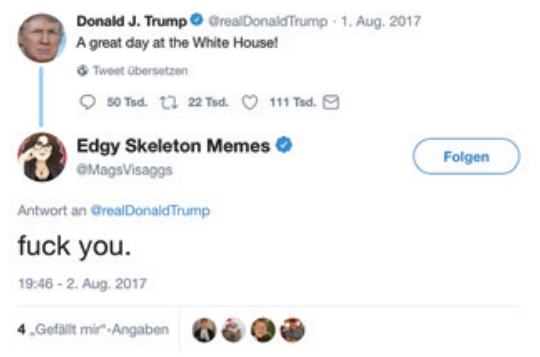

b

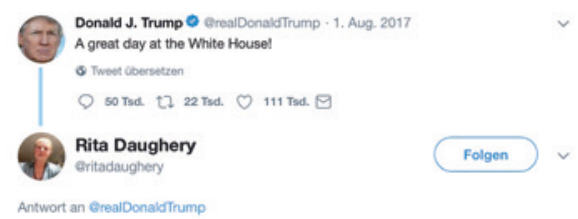

Keep up the great work. The people are with you

Q Tweot abonotan

23:45 - 4. Aug. 2017

Abb. 2 a und b: Tweets von @ MagsVisaggs (02.08.2017) und @ ritadaughery (04.08.2017) 
in Freund und Feind, wie sofort einsichtig wird, wenn man sich die Kommentare zu Trumps Tweets nur einmal anschaut. Die Kommentare zu jedem Tweet Trumps, und sei es ein Ostergruß, aus den letzten drei Jahren belegen, dass Follower auf Twitter nicht mit einem affirmativen Gefolge des Präsidenten zu verwechseln sind. Von einem „Phantasma der Metonymie des ,Volkes“ der Follower mit seinem populistischen Führer" kann man nur sprechen (ebd.: 72), wenn man die Medienpraktiken des Twitterns vollkommen ignoriert. Was sich auf dem Account @ realDonaldTrump konstituiert, ist alles andere als eine homogene Volksgemeinschaft, die in Trump ihren wahren Repräsentanten gefunden hat. So wie es bis heute keinen dislike-Button gibt und in den ersten sechs Jahren der großen Twitter-Karriere Trumps noch nicht einmal einen like-Button (Paßmann 2018), so teilt sich auch die politische Welt Trumps auf gar keinen Fall in „Follower und alle anderen“, so als seien die Follower Freunde und die anderen Feinde. Es ist vielmehr so, dass alle Follower, seien sie nun Fans oder Hater, Trump nolens volens gemeinsam populär machen, während alle anderen dies (bislang) nicht tun. All jene, die nicht twittern oder seinen Account nicht abonnieren, sind für Trump entsprechend potenzielle Follower, die im Dienste der Popularität noch inkludiert werden müssen.

Nach dieser Kritik sollen im Folgenden Trumps Twitter-Praktiken genauer beschrieben werden. Um die Spezifität dieser medialen und politischen Praxis genauer in den Blick zu bekommen, nutze ich als Kontrastfolie Erwartungen, die aus der Epoche der ausschließlich massenmedialen Reflexion der Politik stammen, also aus der Zeit vor der Popularisierung des Internets.

\section{Trumping Twitter}

In den soziologischen Großtheorien von Jürgen Habermas und Niklas Luhmann spielen die alten Massenmedien eine zentrale Rolle für repräsentative Demokratien: Die „öffentliche Meinung“ übernehme für die Politik der Gesellschaft die Funktion eines „Mediums für ein Beobachten zweiter Ordnung“ (Luhmann 2000: 287). Die Politik kann hier beobachten, wie sie beobachtet wird - und dies nicht im Modus einer Interaktion unter Anwesenden, sondern mit Bezug auf ,Darstellungen abwesender Beobachter für abwesende Beobachter" (ebd.: 295), also in Radio, Presse und Fernsehen (Luhmann 1996: 11). Die Massenmedien dienen der „Repräsentation von Öffentlichkeit“ (ebd.: 188) in dem Sinn, dass die Politik sich selbst mit Blick auf ihre vermeintliche Wirkung auf die ,öffentliche Meinung ‘ zu reflektieren vermag. Luhmann geht in seinen Monografien zum Thema davon aus, dass dies alle Akteure wissen und sich entsprechend auf die Beobachtung durch den Anderen einstellen (können). Für die Politikerinnen und Politiker führe dies zum Bemühen um „Ausdrucksbeherrschung und Informationskontrolle“ (Luhmann 2010: 292). Oder abstrakter formuliert: „Daß alle Äußerungen, die sich im Medium der öffentlichen Meinung präsentieren, sich eben damit selbst der Beobachtung aussetzen, hat disziplinierende Effekte zur Folge“ (Luhmann 2000: 291). Mit dieser Konzeption der öffentlichen Meinung ist der Rahmen skizziert, den Trump in mehrfacher Hinsicht bricht. 
Zum ersten: Die Massenmedien werden in eine parasitäre Rolle gedrängt, denn Trump unterläuft die von „Organisationen strukturierte Öffentlichkeit“ (Luhmann 2010: 434) mit jedem seiner bislang 39.800 Tweets, mit denen er inzwischen Hunderte von Millionen Usern erreicht, nämlich seine 55 Mio. Follower und in einem Kaskadeneffekt auch die Follower seiner Follower, die ihn liken oder retweeten. Wenn die Massenmedien über die Tweets berichten, popularisieren sie zugleich den Account.

Zweitens: Der twitternde Trump macht sich von allen modernen, bürokratisch-administrativen Filtern unabhängig, die traditionell zwischen der Meinung eines gewählten Präsidenten und der veröffentlichen Meinung seiner Regierung stehen. Der Zugang zum Präsidenten wurde in den USA im Verlauf des 20. Jahrhunderts drastisch verknappt. Bereits zu Beginn des Jahrhunderts belegen Zeugnisse führender Journalisten und Publizisten die Verwunderung darüber, keinerlei Informationen mehr vom Präsidenten zu erhalten und ihm ihrerseits keine Hinweise oder auch keinen Rat mehr geben zu können (Nelson 2000: 21 f.). Franklin D. Roosevelts Sekretär Stephen Early führt in den 1930er Jahren regelmäßige Presse-Briefings ein, die vom White House Press Secretary organisiert werden. Seitdem spricht und schweigt der Press Secretary für den Präsidenten, und er simuliert und er dissimuliert zu dessen Vorteil und Schutz, während der Präsident selbst je nach Lage davon absehen kann, zu sprechen oder zu lügen, zu leugnen oder auszuweichen. In der US-Publizistik wird das Dilemma des White House Press Secretary betont, der oder die (Dana Parino übernimmt 2007 als erste Frau dieses Amt, das zur Zeit Sarah Sanders innehat) einerseits die Öffentlichkeit in Gestalt des Pressecorps wahrheitsgemäß zu informieren hat und anderseits der Beliebtheit und Popularität des Präsidenten bzw. dessen Schutz vor einer stets neugierigen und zudringlichen Öffentlichkeit verpflichtet ist (Klein 2008). Working the press, spinning oder hiding news, wie immer man nun die Aufgaben dieses Amtes auch interpretieren mag, klar ist jedenfalls, dass eine Agentur eingerichtet worden ist, die im White House die Kommunikation ,zwischen Politik und Publikum“ organisiert, verwaltet und betreut (hat?) (Luhmann 2010: 292). Und noch für Barack Obama war diese Praxis ganz selbstverständlich, auch was die Handhabung seines enorm populären Twitter-Accounts angeht, dem als Präsident über 100 Mio. Accounts folgten: „Barack Obama was a reluctant pioneer in this regard. Tweets from his @POTUS account would undergo a policy and legal vetting process, and it could sometimes take days or even weeks before they were finally posted" (Draper 2018). Der 44. Präsident der Vereinigten Staaten wendet sich auf Twitter keinesfalls , direkt' oder, unmittelbar' an sein Publikum (Strohschneider 2018: 61), sondern mediatisiert, wie es Luhmann für typisch hält.

Die Verlautbarungen Obamas kommunizieren nicht mit, dass ihnen ein ,policy and legal vetting process" vorausgegangen sind; und auch ein Pressesprecher expliziert es üblicherweise nicht, wenn sein briefing in Wahrheit ein spinning ist. Würde die Person darauf hinweisen, dass ihre Verlautbarungen fabriziert oder wenigstens selektiert und inszeniert oder auch Ablenkungen und Ausflüchte sind, gäbe es Irritationen, die auf einen Bruch des Rahmens hinweisen würden, in dem das White House Press Corps und der White House Press Secretary gemeinsam zu 
agieren vorgeben. Wenn Trumps zeitweiliger Pressesprecher Sean Spicer wissen lässt, er würde nicht nur die Politik seines Präsidenten vermitteln, ,but to make false statements, if asked to do so by the president" (Borchers 2017), wird diese Geschäftsgrundlage zerstört.

Trump kommuniziert nun aber selbst mit seinem Publikum, und zwar täglich, und Kommunikationsdirektoren wie Scaramucci benötigt er dafür nicht. Trump verzichtet auf die Dienste einer Instanz, die üblicherweise verhindern soll, dass Differenzen zwischen der Darstellung seines Handelns und dem Kalkül seines Handelns, zwischen der Mitteilung einer Information und ihrer Motivation an ihm selbst beobachtet werden können. Trump dagegen bricht mit dieser Praxis, ruiniert das Amt des Sprechers und Kommunikationsdirektors gründlich und macht sich selbst Tweet für Tweet beobachtbar - und kann daher persönlich des Lügens und Leugnens, der Simulation und Dissimulation bezichtigt werden. Seine Sprecher und Sprecherinnen haben darauf bislang recht hilflos reagiert und vergeblich versucht, die Verlautbarungen ihres Präsidenten einzuhegen, aus denen die New York Times eine lange Liste zusammengestellt hat mit „nearly every outright lie he has told publicly since taking the oath of office" (Leonhardt/Thompson 2017). Da die Sprecherinnen und Sprecher nicht wissen, wozu und wie ihr Präsident sich äußern wird, verfügt das White House Press Corps oft genug über die gleichen Informationen wie Spicer und Scaramucci oder Sanders und Hicks, die auch nur Trumps Tweets zur Kenntnis nehmen können. An dieser Informationslage ändert sich auch nichts, wenn Robert Draper recht hat und einige Tweets Trumps von seinem Social Media Director Dan Scavino geschrieben worden sind (Draper 2018), denn auch über diese Tweets haben Sanders und Hicks vorab keine intimeren Kenntnisse als das Press Corps. Kein Wunder, dass der Account @PressSec vor allem für Retweets der Tweets von @ realDonaldTrump benutzt wird. Am 12.05.2017 twittert Trump unvermittelt und im dargelegten Sinne durchaus konsequent: „... Maybe the best thing to do would be to cancel all future ,press briefings' [...].“

Es gibt noch ein weiteres Motiv, das Trump auf den Gedanken bringt, das White House Press Corps aufzulösen und nach Hause zu schicken; und auch dieses Motiv wird völlig unverhohlen und unvermittelt, also ohne jedes Bemühen um die von Luhmann unterstellte „Ausdrucksbeherrschung und Informationskontrolle" benannt: Trump gefällt es nicht, wie die alten Medien über ihn berichten. Über hundert Mal hat Trump auf Twitter die seriösesten Massenmedien wie CNN und Washington Post, ABC, NBC und New York Times als Fake News denunziert. Auf ein ,totally biased and dishonest Media coverage“ (22.09.2017) könne er auch verzichten. In den liberalen Medien wie etwa Politico, Huffington Post, Newsweek, NY Post usw. spricht man von „Trump's war on press“; in den Alt-Right-Medien wird dieser Krieg begrüßt, gefördert und gefeiert. Tatsächlich wird die Rolle der Massenmedien für einen öffentlichen, kritischen Diskurs über die Politik des Präsidenten genauso marginalisiert wie seine Pressesprecherinnen, wenn Trump sich lieber via Twitter ,direkt" an seine Follower wendet, statt die Vermittlung den Fake News Media zu überlassen: 
[TRUMP:] „Well, let me tell you about Twitter. I think that maybe I wouldn't be here if it wasn't for Twitter, because I get such a fake press, such a dishonest press... They're despicable in their coverage. CBS, ABC, you take a look at what's going on - I call it the fake press, the fake media. It is a disgrace" (Cillizza 2017).

Drittens: Von einer „Repräsentation von Öffentlichkeit“ (Luhmann 1996: 188) im Sinne von Habermas und Luhmann kann gar keine Rede sein, wenn Trump seine Verlautbarungen ohne Umwege über Presseamt und Pressekorps publik macht. Ohne Mediatisierung und Moderierung durch seine angestellten Sprecherinnen oder offiziellen Berater exponiert sich Trump in einer in fast jeder Hinsicht unkontrollierbaren Weise. Hunderttausende von Kommentaren seiner Tweets beziehen sich völlig unvermittelt auf Trump als Person. Die Sachdimension seiner Tweets erzeugt kaum Resonanz, auffällig ist die Begeisterung über Trump oder seine entschiedene Ablehnung als Präsident und - vor allem - als Mensch.

Ein typisches Beispiel für die Affirmation Trumps auf Twitter ist ein Tweet einer Evelyn McQuary vom 4. Oktober 2017, für den sich Trump am 05.10. mit einem schlichten wie pathetischen „Thank you“ bedankt hat: „God bless President Trump, his family and his team who are faithfully and diligently MAKING AMERICA GREAT AGAIN!" Der Account ist übrigens ein Bot. Dass sich Trump bei einem Bot bedankt, ist aber durchaus angemessen, schließlich wird er von Abertausenden von Fake Followers unterstützt. Aber auch ganz ohne Bots erfährt Trump auf Twitter im Minutentakt Zustimmung: Make America Great Again! \#MAGA.

Charakteristisch für die Ablehnung Trumps ist ein Tweet, der den Präsidenten als „pathological liar“ und „fucking moron“ bezeichnet (s. Abb. 3). Das klingt in den Kommentaren von Trumps Tweets auf Twitter dann auch ganz anders als in Briefen an den Editor der Times oder Post: Mit "fucking liar" geht es los, tausendfach, und mit dem Aufruf „assassinate Trump“ hört es nicht auf. Neben Obszönitäten, Verwünschungen, Drohungen und Mordaufrufen der Trump-Feinde stehen die Liebes- und Ergebenheitsadressen seiner Fans, die ihn vergöttern und seine Kritikerïnnen verwünschen und bedrohen. Dass Trump seinerseits Personen,

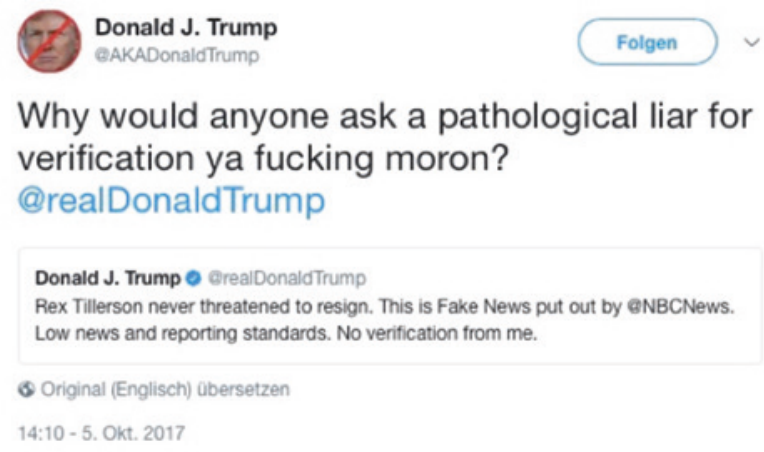

Abb. 3 Tweet von @AKADonaldTrump (05.10.2017) 
Organisationen, Medien harsch attackiert, gibt dieser Spirale ein weiteres Momentum. Dies ist mein vierter Punkt: Twitter eskaliert. Die devote Zustimmung und der ungezügelte Hass seiner Follower, Bots auf beiden Seiten inklusive, erzeugen jene Resonanz, die Trumps Account so „super strong“ machen. Die Verbalinjurie „fuck you“ wird in den Kommentaren der Tweets Trumps den Feinden des Präsidenten genauso wie seinen Fans entgegengeschleudert. Es ist das inoffizielle Motto der 45. Präsidentschaft. Zum einen symbolisiert diese Invektive die für Trumps Tweets typische Geste, auf Verfassung, Bundesstaaten, Richter, Parteien, Unternehmen, Medien, Bündnisse, Verträge, Institutionen keinerlei Rücksicht zu nehmen. Trump lässt alle seine Follower, Anhängerïnnen wie Gegnerïnnen, spüren, dass sie und er wissen, dass er ,darübersteht. Damit markiert er eine übergeordnete Souveränität" - eine typische Medienpraktik des Twitterns (Paßmann 2017: 337). Zum anderen zeigt die Ubiquität dieser Beleidigung auf dem @ realDonaldTrump-Account, dass alles Politische persönlich wird: Die Auseinandersetzung wird nicht mit Blick auf den Sachgehalt einer Meinung geführt, sondern mit dem Ziel der Diffamierung des Anderen dafür, dass er anderer Meinung ist als der Präsident. Anti-Trump - fuck you! Oder: Anti-anti-Trump - fuck you! Mit jedem seiner Tweets erzeugt Trump so die enorme Resonanz seines Accounts zugleich durch Ablehnung und durch Zustimmung! Dies gilt gerade auch im Vergleich zu @potus, auf dem die Beteiligung an dieser Eskalation deutlich schwächer ist. Auch diese Polarisierung verschafft Trump das, was ihm am allerwichtigsten ist: Popularität. Diese Priorität ist von seinem ersten Tweet an offensichtlich und zieht sich als roter Faden durch seine Timeline. Am 21.09.2017 retweetet@ @realDonalTtrump (05:30:56) eine Twitter-Statistik, der zufolge Trump auf Platz 1 der World Leader stehe - und zwar nicht etwa, was die Beliebtheit oder Bedeutung betrifft, sondern schlicht was seine Beachtung in Zahlen angeht: Über ihn wird am meisten getwittert. In dieses Ranking gehen alle „hater“, „,snowflakes" und sonstigen ,loser" mit ein, die Trump nur thematisieren, um ihn zu beleidigen oder zu kritisieren. Feinde und Freunde machen den Account gemeinsam stark; in diesem Sinne hat Trump ganz Amerika vereint: Jeder einzelne Tweet von Trump aus den letzten drei Jahren genügt, damit sich Tausende gegenseitig als Rassisten, Nazis, Idioten, Lügner oder Verräter beschimpfen. Man würde an keiner Stelle auf den Gedanken kommen, dass in diesem öffentlichen Medium die „Publizität als Prinzip der Vermittlung von Politik und Moral“ wirksam sein könnte, wie Immanuel Kant und Jürgen Habermas dies vom öffentlichen Diskurs angenommen haben (Habermas 1990: 178 ff.; Kant 1984). Dass ein politisches Vorhaben oder eine politische Handlungsmaxime „öffentlich kundbar“ ist, liefert für Kant ein sicheres Kriterium dafür, dass die entsprechende politische Agenda auch „ethisch“ und ,juridisch“ gerechtfertigt sei; man könne sich dazu „öffentlich bekennen“, „ohne daß dadurch unausbleiblich der Widerstand aller gegen meinen Vorsatz gereizt werde“ (Kant 1984: 49 f.). Diese ,politisch fungierende Öffentlichkeit“" avanciert in Habermas' berühmter Studie zum Strukturwandel der Öffentlichkeit zum „Organisationsprinzip des liberalen Rechtsstaates“ (Habermas 1990: 183). Trumps Tweets erweisen das Gegenteil: sie reizen unausweichlich zum Widerstand und zum Widerstand gegen diesen Widerstand, sodass sich am Ende 
nur noch der Hass der Trump Lover gegen die Trump Hater zu neuen Bosheiten und Verleumdungen aufzuschaukeln scheint und alles, was man in Trumps Tweets überhaupt als konkretes politisches Vorhaben oder allgemeine politische Maxime entziffern könnte, im Resonanzraum der Twitter-Kommentare keine Rolle mehr spielt.

Fünftens: Es ist vor Trump nicht üblich gewesen, politische Entscheidungen primär an ihrer Popularität auszurichten und dies auch mitzuteilen. Luhmanns Feststellung, dass in der Politik die „Darstellung [...] kalkuliert, aber die Kalkulation nicht dargestellt werden darf" (Luhmann 2010: 292), wird von Trump auf den Kopf gestellt, denn er möchte nicht allein an einer Sachfrage, sondern vor allem an der Beachtung gemessen werden, die ihm selbst zukommt, wenn er sich einer Sache annimmt. Diese Orientierung an der Popularität in Form messbarer Beachtung - zumal durch Millionen von Follower oder fahnenschwenkenden Fans - legt einen Politikstil nahe, den man occasionalistisch nennen könnte. Selbstbindungen, die Trump hindern könnten, eine Gelegenheit zu ergreifen, lehnt er dezidiert ab. Am 18.12.2012 twittert er programmatisch: „I also protect myself by being flexible. I never get too attached to one deal or one approach. - The Art of The Deal".

Ein Beispiel für diesen Occasionalismus, der sich keine Gelegenheit zur Popularisierung entgehen lässt, lieferte seine harsche Polemik gegen einige schwarze NFL-Spieler. Trump hat einem NFL-Spiel zugesehen und bemerkt, dass sich einige Spieler zur Nationalhymne nicht erhoben haben. Auf Twitter fordert er, während das Spiel noch läuft, die Football-Spieler, die Millionen verdienen, „should stand for the National Anthem. If not, YOU'RE FIRED“ (23.09.2017). YOU'RE FIRED gehört zur Marke Trump, und er wiederholt diesen Spruch aus seiner TV-Show The Apprentice (NBC seit 2004) immer wieder, wenn es sich anbietet. Trump weiß, was seine Follower von ihm erwarten, die ihn als den „,you're fired" president“ feiern (retweet am 05.10.2016). Popularisiert wird, auch was das politische Problem der NFL-Spieler angeht, nicht die Sachfrage oder eine politische Kontroverse, sondern die Geste selbst. Der tautologische Gipfel - you're fired because I am a „you're fired“ president - wurde beinahe erreicht, als Trump seinen Kommunikationsdirektor Anthony Scaramucci nach nur zehn Tagen im Amt entließ und twitterte: „A great day at the White House“ (31.07.2017)!

Ein Sechster Punkt kommt hinzu: Am 25.08.2017 teilt der Autor und Trump-Kritiker Stephen King mit, Donald Trump habe ihn auf Twitter geblockt, er könne also dem Account nicht länger folgen, keinen der Tweets einsehen oder kommentieren. King und viele andere werden aus dem bevorzugten Nachrichtenkanal des Präsidenten ausgeschlossen. Die gegen diese Praxis Trumps vor dem United States District Court geführte Prozess ist zwar am 23. Mai 2018 von der Richterin Naomi Reice Buchwald zugunsten der Kläger entschieden worden, ${ }^{2}$

\footnotetext{
${ }^{2}$ Das Urteil im Original als PDF: https://knightcolumbia.org/sites/default/files/content/Cases/ Wikimedia/2018.05.23\%20Order\%20on\%20motions\%20for\%20summary\%20judgment.pdf (24.04.2019).
} 
doch hatte diese Entscheidung keinerlei Folgen. Die Erwartung des Gerichts, ,we must assume that the President and Scavino will remedy the blocking we have held to be unconstitutional“, wird bislang enttäuscht. Auf @ realDonaldTrump wird weiterhin geblockt.

Eine solche Exklusionsmöglichkeit bieten die Massenmedien nicht. Die ,öffentliche Meinung' demokratischer Gesellschaften ist juristisch gemeinhin gegen solche Ausschlüsse geschützt. Niemandem kann verboten werden, Zeitungen zu lesen, die von allen anderen Bürgerinnen und Bürgern gelesen werden, oder Sendungen zu sehen, die von jeder Person empfangen werden können. „Im klassischen [...] Diskurs“, darauf weist Niklas Luhmann hin, sei „,öffentlich“ durch Zugänglichkeit für jedermann, also durch Ausschluß der Kontrolle über den Zugang definiert. Danach sind Druckerzeugnisse und Sendungen der Massenmedien öffentlich, weil keine Kontrolle darüber besteht, wer sie zur Kenntnis nimmt“ (Luhmann 1996: 184). Auch dieser Zusammenhang von Öffentlichkeit und Massenmedien wird von Trump gesprengt, denn zum einen liegt die Kontrolle des Zugangs zu einem Twitter-Account bei seinem Betreiber, der unliebsame User blockieren, stumm stellen oder sperren kann, und zum anderen herrscht für alle vollkommene Transparenz darüber, wer folgt, wer retweetet, wer liked, wer kommentiert. Wer seine Tweets wie zur Kenntnis nimmt, ist Trump prinzipiell bekannt. Unter dieser Voraussetzung blockiert er ja seine schärfsten und populärsten Gegner.

Das von Luhmann angeführte doppelte Kontrollverbot - Ausschluss der Kontrolle über den Zugang zur einer Nachricht und keine Kontrolle darüber, wer sie zur Kenntnis nimmt - gilt für Twitter grundsätzlich nicht; man könnte eher im Gegenteil von den ungeheuren Möglichkeiten dieses Mediums sprechen, Rezeption wie Produktion zu einer höchstpersönlichen, individualisierten Sache zu machen. Statt ohne Ansehen der Person geht es bei Twitter um das Gegenteil: Gerade das Ansehen der Person, ja eigentlich das Selbst des Individuums stehen auf dem Spiel. Zorn und Eifer eskalieren noch die Kommentare des harmlosesten Tweets, etwa des Ostergrußes vom 16.04.2017, zwischen „I love you“ und „Even Jesus hates you“, „God bless you“ und „suk me“, „,impeach Trump“ und „MAGA“.

Die Kommentare eines Trump-Tweets sehen anders aus als die Leserbriefseiten der Qualitätspresse. Auf Twitter wird alles persönlich genommen, also Mensch und Amt, Individuum und Person, Subjekt und Rolle werden nicht getrennt, wie dies in der modernen, funktional ausdifferenzierten Welt der Normalfall sein soll, sondern jede Äußerung mit der Achtung oder Missachtung des ,ganzen Menschen" verbunden - als ob es um eine moralische Interaktion unter Anwesenden ginge. Dies gilt auch für Trumps eigene Tweets, die notorisch die moralische Verkommenheit seiner Kritikerinnen und Kritiker bedauern. „Sad! So sad!“.

Die Moral, die sich in der Moderne aus dem politischen Raum zurückgezogen hat, ist - siebtens - zurück, was keine gute Nachricht ist, denn die Moralisierung von politischen Unterscheidungen zeitigt polemische Effekte: Die Anderen vertreten nicht nur eine andere Meinung, die man ja mit guten Gründen ablehnen und 
politisch bekämpfen kann, sondern sind als Menschen böse, verdorben, schlecht. Diese Anderen müssen gehasst werden. Diese Moralisierung der politischen Auseinandersetzung bis hin zur Ächtung ist paradigmatisch abzulesen an dem Hillary Clinton kriminalisierenden Hashtag \#LockHerUp. Die Konkurrentin „Crooked Hillary“ (404 mal) wird zu einem Feind und Verbrecher (s. Abb. 4). „Moral“ sei, so Luhmann, ,immer ein Symptom für das Auftreten von Pathologien“ (ebd.:143), und im Fall der moralischen Zuspitzung der politischen Debatte zum Twitter-Krieg wäre eine Pathologie der Politik der Gesellschaft zu indizieren: Der Rahmen, den die repräsentative, auf öffentlichem Diskurs aufbauende, liberale Demokratie benötigt, ist kollabiert.

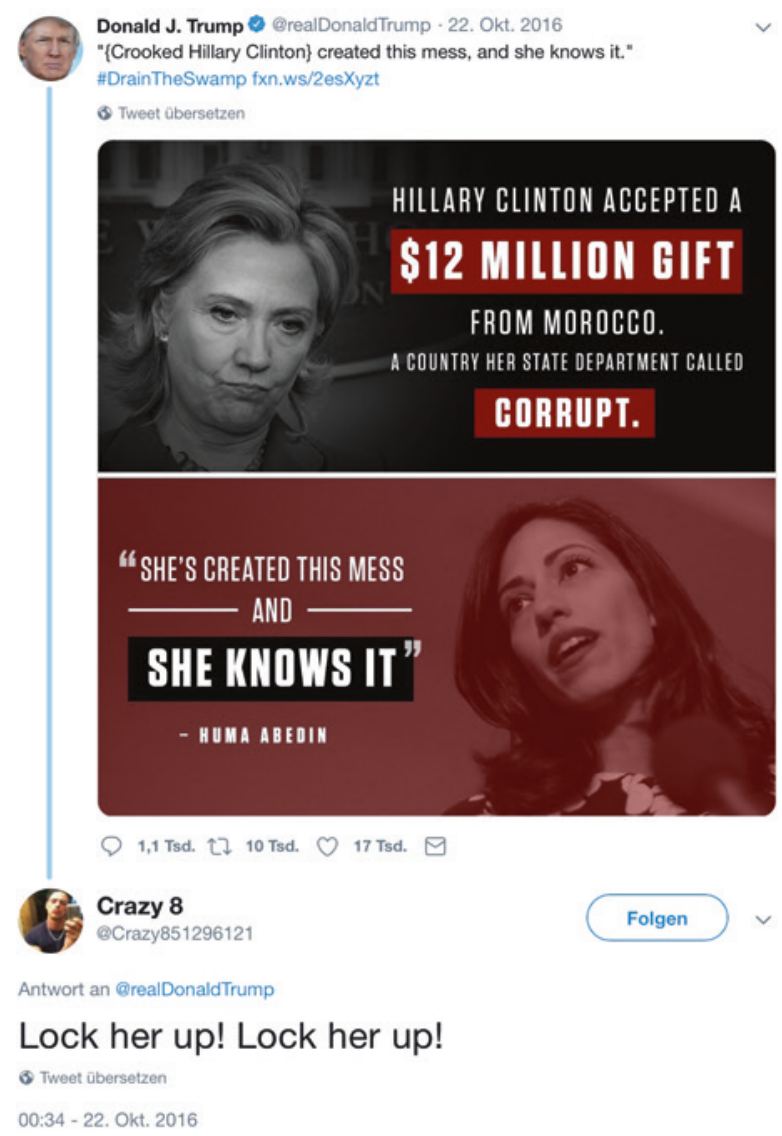

Abb. 4 Tweets von @ realDonaldTrump und @crazy851296121 (22.10.2016) 


\section{New normal}

Als vorübergehendes Störmoment oder Krisenereignis, das mit der nächsten oder doch übernächsten Wahl verschwände, wäre Trump missverstanden, weil seine Medienpraktiken einen neuen Rahmen etabliert haben, in dem seine Tweets nicht als Ausnahme zu betrachten sind, sondern als erwartbar. Dass ein Tweet inkohärent ist, nicht der Wahrheit entspricht oder in Widerspruch mit einem anderen Tweet Trumps gerät - all dies zählt zum new normal einer occasionalistischen, allein an Popularität orientierten Medienpraxis, für die es auf die Steigerung der Beachtung ankommt, nicht aber auf Konsistenz mit der Vergangenheit. ${ }^{3}$ In diesem neuen Rahmen spielen Gesetze und Verträge, Institutionen, Programme und Normen eine untergeordnete Rolle, da sie kollektiv bindende Entscheidungen oder Präferenzen über die Zeit hinweg festhalten und so auch zukünftiges Handeln an die Vergangenheit binden. Genau dieser Rahmen wurde aber zerschlagen. Daher ist Trump mit dem Vorwurf der Lüge gar nicht beizukommen, da das Konzept der Lüge einen kausalen und temporalen Zusammenhang voraussetzt, den es in der Gegenwärtigkeit der deals nicht gibt, weil auch der deal nur die occasio völlig opportunistisch nutzt - sich also um vergangene Festlegungen nicht kümmert. Die penible Dokumentation aller Lügen durch die New York Times geht so an der Wirklichkeit, die Trump auf Twitter organisiert, völlig vorbei.

Trump agiert in einer Gegenwart, in der ihm alles zur Gelegenheit für einen Tweet werden kann. Dass dieser Tweet die Popularität des Präsidenten erhöht, liegt im Mechanismus der Beachtungserzeugung begründet, den Trump mit Twitter dadurch perfektioniert hat, dass seine Befürworterïnnen und seine Gegnerïnnen, seine Fans und seine Kritikerïnnen die politische Auseinandersetzung zu einem Krieg eskaliert haben, der dem Account @ realDonaldTrump täglich ca. 70.000 neue Follower beschert, die sich gegenseitig beschimpfen, beleidigen und bedrohen und alle gemeinsam die Resonanz des Accounts weiter erhöhen. Auf der Twitter analytics service-Plattform Twitter Counter ist dazu zu lesen: „That's awesome! @ realDonaldTrump seems to be on its way to Twitterverse domination!“ Die unverhohlene Freude über die Popularität des Accounts, der zugleich die Popularität der Plattform indiziert, kann nun nicht mehr verblüffen und belegt noch einmal das Primat der Popularisierung der Trumpschen Twitter-Praxis.

Selbstverständlich kann man dies alles Populismus nennen. Aber dann ist der Populismus Trumps vor allem eine Bezeichnung für äußerst Populäres, dessen Popularität man ablehnt - als vulgär zum Beispiel, als unsäglich (Strohschneider 2018: 62), als irrational oder unmenschlich (vgl. Manow 2018a, b). Normativ steht man dann auf der sicheren Seite, auf der Seite, die nicht populistisch ist, auf der Seite der Vernunft und Weitsicht, der Argumente und der Menschlichkeit. Einer Analyse der Popularisierungsbedingungen des Populismus kommt man so allerdings nicht näher. Ich möchte daher dafür plädieren, die Erarbeitung

\footnotetext{
${ }^{3}$ Ähnlich argumentiert hier Strohschneider 2018: 68. Vgl. zum Ersetzen der causa durch die occasio Werber 2017.
} 
von Populismus-Definitionen nicht mit der Analyse des populistischen Feldes zu verwechseln. Die Medienpraktiken besser zu verstehen, die der Popularisierung dienen, scheint mir ein wichtiger Schritt auf dem Weg zum Verständnis einer Popularität zu sein, die die Rahmenbedingungen der repräsentativen, in der Öffentlichkeit reflektierten, liberalen Demokratie auf den Kopf stellt.

\section{Literatur}

Berke, Jon: A trip down Donald Trump's bankruptcy memory lane. In: Forbes (18.08.2015), https://www.forbes.com/sites/debtwire/2015/08/18/a-trip-down-donald-trumps-bankruptcymemory-lane/\#5923f214609e (11.03.2019).

Borchers, Callum: Sean Spicer basically admitted that he was willing to lie for Trump. In: The Washington Post (14.09.2017), https://www.washingtonpost.com/news/the-fix/ $\mathrm{wp} / 2017 / 09 / 14 /$ sean-spicer-basically-admitted-that-he-was-willing-to-lie-for-trump/?utm _ term $=.2 \mathrm{~d} 81991 \mathrm{ba0b} 4(11.03 .2019)$.

Cillizza, Chris: Donald Trump's explanation of his wire-tapping tweets will shock and amaze you. In: The Washington Post (16.03.2017), https://www.washingtonpost.com/news/the-fix/ $\mathrm{wp} / 2017 / 03 / 16 /$ donald-trump-explained-twitter-the-universe-and-everything-to-tucker-carlson/?utm_term=.ca722bf1dc1b (11.03.2019).

Draper, Robert: The man behind the President's tweets. In: The New York Times (16.04.2018), https://www.nytimes.com/2018/04/16/magazine/dan-scavino-the-secretary-of-offense.html (12.04.2019).

Habermas, Jürgen: Strukturwandel der Öffentlichkeit. Untersuchungen zu einer Kategorie der bürgerlichen Gesellschaft [1962]. Frankfurt a. M. 1990.

Hecken, Thomas: Der populäre Donald Trump. In: Pop. Kultur und Kritik 10 (2017): 10-21.

Hecken, Thomas: Populäre Kultur. Mit einem Anhang , Girl und Popkultur'. Bochum 2006.

Heintz, Bettina: Von der Allmacht der Zahlen und der Allgegenwart des Bewertens. In: Soziologische Revue 41/4 (2018): 629-642.

Kant, Immanuel: Zum ewigen Frieden [1795]. Stuttgart 1984.

Klein, Woody: All the Presidents' Spokesmen: Spinning the News, White House Press Secretaries from Franklin D. Roosevelt to George W. Bush. Westport, Connecticut 2008.

Leonhardt, David/Thompson, Stuart A.: Trump's lies. In: The New York Times (14.12.2017), https://www.nytimes.com/interactive/2017/06/23/opinion/trumps-lies.html?mcubz=3 (11.03.2019).

Luhmann, Niklas: Politische Soziologie, hg. von André Kieserling. Berlin 2010.

Luhmann, Niklas: Die Politik der Gesellschaft. Frankfurt a. M. 2000.

Luhmann, Niklas: Die Realität der Massenmedien. 2. Aufl. Opladen 1996.

Manow, Philip: „Dann wählen wir uns ein anderes Volk..." Populisten vs. Elite, Elite vs. Populisten. In: Merkur. Deutsche Zeitschrift für europäisches Denken 74/4 (2018a): 5-14.

Manow, Philip: Die Politische Ökonomie des Populismus. Berlin 2018b.

Mau, Steffen: Das metrische Wir. Über die Quantifizierung des Sozialen. Berlin 2017.

Müller, Jan-Werner: Populismus: Theorie und Praxis. In: Merkur. Deutsche Zeitschrift für europäisches Denken 69/795 (2015): 28-37.

Müller, Jan-Werner: Was ist Populismus? Berlin 2016.

Nelson, W. Dale: Who Speaks for the President? The White House Press Secretary from Cleveland to Clinton. New York 2000.

Paßmann, Johannes: Die soziale Logik des Likes. Eine Twitter-Ethnografie. Frankfurt a. M./New York 2018.

Paßmann, Johannes: Kurz \& souverän. Twittern als sozioliterarische Praxis. In: Michael Gamper/Ruth Mayer (Hg.): Kurz \& knapp. Zur Mediengeschichte kleiner Formen vom 17. Jahrhundert bis zur Gegenwart. Bielefeld 2017: 325-348. 
Schaffrick, Matthias: Listen als populäre Paradigmen. Zur Unterscheidung von Pop und Populärkultur. In: KulturPoetik 16/1 (2016): 109-125.

Strohschneider, Peter: POTUS als Twitterer. In: Zeitschrift für Ideengeschichte XII/3 (2018): $61-75$.

Werber, Niels: Keine Nacht ohne Tweet, kein Tag ohne Dekret. Trumps Show der permanenten Provokation und unaufhörlichen Überschreitung. In: Pop. Kultur und Kritik-Blog, 10.02.2017. http://www.pop-zeitschrift.de/2017/02/10/keine-nacht-ohne-tweet-kein-tag-ohne-dekretvonniels-werber10-2-2017 (01.04.2019).

Open Access Dieses Kapitel wird unter der Creative Commons Namensnennung 4.0 International Lizenz (http://creativecommons.org/licenses/by/4.0/deed.de) veröffentlicht, welche die Nutzung, Vervielfältigung, Bearbeitung, Verbreitung und Wiedergabe in jeglichem Medium und Format erlaubt, sofern Sie den/die ursprünglichen Autor(en) und die Quelle ordnungsgemäß nennen, einen Link zur Creative Commons Lizenz beifügen und angeben, ob Änderungen vorgenommen wurden.

Die in diesem Kapitel enthaltenen Bilder und sonstiges Drittmaterial unterliegen ebenfalls der genannten Creative Commons Lizenz, sofern sich aus der Abbildungslegende nichts anderes ergibt. Sofern das betreffende Material nicht unter der genannten Creative Commons Lizenz steht und die betreffende Handlung nicht nach gesetzlichen Vorschriften erlaubt ist, ist für die oben aufgeführten Weiterverwendungen des Materials die Einwilligung des jeweiligen Rechteinhabers einzuholen. 